\title{
Dense 2D Displacement Reconstruction from SPAMM-MRI with Constrained Elastic Splines: Implementation and Validation ${ }^{1}$
}

\author{
Amir A. Amini ${ }^{1}$, Yasheng Chen ${ }^{1}$, Jean Sun ${ }^{1}$, and Vaidy Mani ${ }^{2}$ \\ 1 CVIA Lab, Box 8086, 660 S. Euclid, \\ Washington University Medical Center, St. Louis, MO 63110 \\ email: amini@mobius . wust1.edu \\ home page: http://ww-cv.wustl.edu \\ 2 Iterated Systems, Inc., Atlanta, Georgia
}

\begin{abstract}
Efficient constrained thin-plate spline warps are proposed in this paper which can warp an area in the plane such that two embedded snake grids obtained from two SPAMM frames are brought into registration, interpolating a dense displacement vector field. The reconstructed vector field adheres to the known displacement information at the intersections, forces corresponding snakes to be warped into one another, and for all other points in the myocardium, where no information is available, a $C^{1}$ continuous vector field is interpolated. The formalism proposed in this paper improves on our previous variational-based implementation and generalizes warp methods to include biologically relevant contiguous open curves, in addition to standard landmark points. The method has been extensively validated with a cardiac motion simulator, in addition to in-vivo tagging data sets.
\end{abstract}

\section{Introduction}

MR tagging is an imaging method which offers an excellent technique for measuring non-rigid motion of the myocardium. With this method, the magnetization property of selective material points are altered in order to create dark stripe patterns within a deforming body such as the heart muscle. The resulting pattern defines a time-varying curvilinear coordinate system on the tissue $[5,15]$, which deforms with the contracting tissue. Tagged MRI, however, is limited in that it can only mark a sparse set of lines and points in the myocardial tissue for tracking of deformations. The problem which we address in this paper is that of reconstruction of dense motion fields from sparse noisy data, not very different from the surface reconstruction problem encountered in stereo vision $[8,12]$. An original solution to the motion reconstruction problem was proposed in $[1,3]$.

${ }^{1}$ Supported in part by a grant from Whitaker Biomedical Engineering Foundation, and grant IRI-9796207 from the National Science Foundation 
However, in this formulation only information at tag crossings were utilized as part of the reconstruction algorithm. In [2], the method was further extended to that of constrained thin-plate reconstruction of the displacement field from points and lines based on a variational solution (for related work, also please see [11].) In this paper, we improve on the reconstruction paradigm in [2] and present extensive validation of the methodologies. One advantage of this line of work is that it allows for reconstruction of dense deformations between 2 arbitrary frames in a sequence of tagged images, as dense motion reconstruction methods generally produce displacement vector fields relative to undeformed tags in the initial frame. (For related work by other researchers please see $[7,9$, 14].)

\section{Constrained Thin-Plate Splines}

Tracking tissue deformations with SPAMM using snake grids provides 2D displacement information at tag intersections and 1D displacement information along other 1D snake points [2]. The displacement measurement from tag lines however are sparse; interpolation is required to reconstruct a dense displacement field from which strain, torsion, and other mechanical indices of function can be computed at all myocardial points. In this section, we describe an efficient solution to the formulation in [2] (this improves on methods in computation time and order of convergence) for reconstructing a dense displacement vector field using localized coordinates of tag positions. In this development, we assume only 2D motion (as is roughly the case towards the apical end of the heart). Although thin-plate warps have been investigated by Bookstein [6], they have been used to interpolate a warp given specified landmarks.

To proceed more formally, the vector field continuity constraint is the bending energy of a thin-plate which is applied to the $x$ and $y$ component of the displacement field $(u(x, y), v(x, y))$ :

$$
\begin{array}{r}
\Phi_{1}=\iint u_{x x}^{2}+2 u_{x y}^{2}+u_{y y}^{2} d x d y+ \\
\iint v_{x x}^{2}+2 v_{x y}^{2}+v_{y y}^{2} d x d y
\end{array}
$$

This serves as the smoothness constraint on the reconstructed vector field, characterizing approximating thin-plate splines.

With intersection "springs" in place, the intersections of two grids are "pulled" towards one another by minimizing

$$
\Phi_{2}=\sum\left(u-u_{i n t}\right)^{2}+\left(v-v_{i n t}\right)^{2}
$$

In (2), $u_{\text {int }}$ and $v_{\text {int }}$ are the $x$ and $y$ components of displacement at tag intersections as well as at intersections of myocardial contours with tag lines. The form of the intersection spring constraints is similar to depth constraints in surface reconstruction from stereo, and has also been used in a similar spirit in [14]. 
Assuming 2D tissue motion, a further physical constraint is necessary: any point on a snake in one frame must be displaced to lie on its corresponding snake in all subsequent frames. This constraint is enforced by introducing a sliding spring. One endpoint of the spring is fixed on a grid line in the first frame, and its other endpoint is allowed to slide along the corresponding snake in the second frame, as a function of iterations. We minimize

$$
\Phi_{3}=\sum\left\{(x+u-\bar{x})^{2}+(y+v-\bar{y})^{2}\right\}
$$

along 1D snake points. In the above equation, $(x, y)$ are the coordinates of a point on the snake in the current frame, and $(\bar{x}, \bar{y})$ is the closest point to $(x+u, y+v)$ on the corresponding snake in the second frame.

\subsection{Conjugate gradient and quasi-Newton algorithms}

Let $(u(x, y), v(x, y))$ be the displacement field as before. The objective function $\Phi(u, v)$ which needs to be minimized is the linear combination

$$
\Phi=\lambda_{1} \Phi_{1}+\lambda_{2} \Phi_{2}+\lambda_{3} \Phi_{3} .
$$

Note that $\bar{x}$ and $\bar{y}$ are dependent on $u$ and $v$ respectively which makes the function $\Phi_{3}(u, v)$ non-quadratic. We can derive the Euler-Lagrange equations for the variational problem in (4) and solve the resulting system of equations [2]. In this paper, we develop a more efficient approach. We follow [8] and straightaway discretize the function $\Phi$ in (4). Assuming the distance between two adjacent grid points to be

$$
u_{i+1, j}-u_{i j}=u_{i, j+1}-u_{i j}=h,
$$

the second order partial derivatives $\left(u_{x x}\right)_{i j},\left(u_{x y}\right)_{i j}$ and $\left(u_{y y}\right)_{i j}$ at the point $(i, j)$ can be approximated by

$$
\begin{aligned}
\left(u_{x x}\right)_{i j} & =\frac{u_{i+1, j}-2 u_{i, j}+u_{i-1, j}}{h^{2}} \\
\left(u_{x y}\right)_{i j} & =\frac{u_{i+1, j+1}-u_{i+1, j}-u_{i, j+1}+u_{i, j}}{h^{2}} \\
\left(u_{y y}\right)_{i j} & =\frac{u_{i, j+1}-2 u_{i, j}+u_{i, j-1}}{h^{2}}
\end{aligned}
$$

The discrete form of the function $\Phi_{1}$ can be obtained by substituting the discrete derivatives into the first equation in (1). The partial derivatives of $\Phi_{1}$ can be calculated using the computational molecule approach discussed in [12] though special attention should be paid in computing the molecules near the endocardial and epicardial boundaries where the smoothness constraint should break in order not to smooth over the motion discontinuity. The endocardial and epicardial boundaries were each manually segmented through-out a slice sequence using a 6 control point cubic B-spline contour. The discretization of the function $\Phi_{2}$ and 
calculation of its partial derivatives is almost trivial. Let us consider the function $\Phi_{3}$ which is non-quadratic. The partial derivatives

$$
\begin{aligned}
\left(\Phi_{3}\right)_{u} & =(u+x-\bar{x})\left(1-\bar{x}_{u}\right)+(v+y-\bar{y})\left(-\bar{y}_{u}\right) \\
\left(\Phi_{3}\right)_{v} & =(v+y-\bar{y})\left(1-\bar{y}_{v}\right)+(u+x-\bar{x})\left(-\bar{x}_{v}\right)
\end{aligned}
$$

For simplification, we now make two approximations. For vertical grids, the $x$-coordinates of curves only vary slightly, and as the grid lines are spatially continuous, $\bar{x}_{u}$ is expected to be small. Furthermore, for vertical grids $\bar{y}$ changes minutely as a function of $u$, so that $\bar{y}_{u} \approx 0$. For horizontal grids, the $y$ coordinates of curves also vary slightly along the length of grid lines, and since these are spatially continuous curves, $\bar{y}_{u}$ is expected to be small. Note that these approximations will hold under smooth local deformations, as is expected in the myocardial tissue. Only $\bar{x}_{u}$ for horizontal grids, and $\bar{y}_{v}$ for vertical grids is expected to vary more significantly. The approximate derivates are now given by:

$$
\begin{aligned}
& \left(\Phi_{3}\right)_{u}=(u+x-\bar{x})\left(1-T_{h o r} \bar{x}_{u}\right) \\
& \left(\Phi_{3}\right)_{v}=(v+y-\bar{y})\left(1-T_{v e r} \bar{y}_{v}\right)
\end{aligned}
$$

The variables $T_{h o r}$ and $T_{v e r}$ are predicates equal to one if the snake point of interest lies on a horizontal, or a vertical grid line. Needless to say, the above functions can be discretized by replacing the continuous values by the corresponding values at the grid points.

After discretization, a typical quadratic optimization problem takes the following form:

$$
f(\mathbf{x})=\mathbf{c}-\mathbf{b}^{T} \mathbf{x}+\frac{1}{2} \mathbf{x}^{T} \mathbf{A} \mathbf{x}
$$

where $\mathbf{x}$ is the vector of variables, $\mathbf{A}$ is the constant Hessian matrix of second order partial derivatives and $\mathbf{b}$ and $\mathbf{c}$ are constant vectors. In the present problem, the terms $\Phi_{1}$ and $\Phi_{2}$ can be cast in the above form. Unfortunately, in the term $\Phi_{3}$, the values $\bar{x}$ and $\bar{y}$ are dependent on $x$ and $y$ respectively which makes $\Phi_{3}$ non-quadratic. The discrete optimization function form of $\Phi$ is given by:

$$
\Phi(\mathbf{x})=\mathbf{c}-\mathbf{b}^{T} \mathbf{x}+\frac{1}{2} \mathbf{x}^{T} \mathbf{A} \mathbf{x}+\lambda_{3} \Phi_{3}(\mathbf{x})
$$

where $\mathbf{A}, \mathbf{b}$ and $\mathbf{c}$ are constants and include the contributions from $\lambda_{1} \Phi_{1}$ and $\lambda_{2} \Phi_{2}$. we are now ready to look at specific minimization algorithms.

The first algorithm we investigate is the conjugate gradient (CG) algorithm. For an order $N$ quadratic problem, the CG algorithm is guaranteed to converge in $N$ iterations. Moreover, it does not store the Hessian matrix and requires $o(N)$ storage for an order $N$ optimization problem. Note that the CG algorithm does not explicitly calculate or store the Hessian matrix $\mathbf{A}$ and can be adapted to the function $\Phi$ in (10). We do not know the Hessian matrix for $\Phi$. However, we do know how to calculate the derivative $\nabla \Phi(\mathbf{p})$ using the derivatives for the functions $\Phi_{1}, \Phi_{2}$ and $\Phi_{3}$. We use this knowledge of the gradient of $\Phi$ in the 
implementation of the CG algorithm. As a final point, since $\Phi$ is non-quadratic, the algorithm may not converge in $N$ iterations. For a description of the CG algorithm please refer to [10].

Quasi-Newton algorithm is a different optimization method that we have investigated. It differs from $\mathrm{CG}$ in that it has higher memory requirements but better convergence properties for non-quadratic functions. By quasi-Newton method we mean techniques which use an approximation to the inverse Hessian matrix in each iteration as opposed to Newton methods which use the exact inverse. A generic quasi-Newton algorithm calculates and stores an approximation to the inverse Hessian matrix in each iteration. Hence for an order $N$ optimization problem, this method needs $o\left(N^{2}\right)$ storage. The advantage of a quasi-Newton algorithm lies in that it has quadratic convergence properties for general smooth functions (not necessarily quadratic). A specific quasi-Newton algorithm is characterized by the approximation it uses for the Hessian matrix. The quasi-Newton method used in this paper is called the Davidon-Fletcher-Powell (DFP) algorithm. An overview of which is given in the [10].

\subsection{Validations}

\section{Cardiac Simulator}

To test and validate the algorithm an environment based on a 13 parameter kinematic model of Arts et al. [4] has been implemented as described in [13] for simulating a time sequence of tagged MR images.

The motion model involves application of a cascade of linear transformations describing rigid (rotations and translations) as well as non-rigid transformations (radial compression, torsion, ellipticalization in SA and LA, as well as shear along $x, y$, and $z$ axes). Once a chosen discretization step is assumed and a mesh for tessellating the 3D space is generated, the linear matrix transformations are applied in a sequence to all the mesh points so as to deform the reference model. The parameters of the motion model, referred to as $\mathrm{k}$-parameters, and the transformations to which they correspond are as follows: 1) $k_{1}$ : Radially dependent compression, 2) $k_{2}$ : Left ventricular torsion 3) $k_{3}$ : Ellipticalization in long-axis (LA) planes 4) $k_{4}$ : Ellipticalization in short-axis (SA) planes 5) $k_{5}$ : Shear in $\mathrm{x}$ direction 6) $k_{6}$ : Shear in y direction 7) $k_{7}$ : Shear in z direction 8) $k_{8}$ : Rotation about x-axis 9) $k_{9}$ : Rotation about y-axis 10) $k_{10}$ : Rotation about zaxis 11) $k_{11}$ : Translation in $\mathrm{x}$ direction 12) $k_{12}$ : Translation in y direction 13) $k_{13}$ : Translation in $z$ direction In order to simulate MR images, a plane intersecting the geometric model is selected, and tagged spin-echo imaging equations are applied for simulating the imaging process.

For the purposes of validating 2D displacement field reconstructions, we have used the parameters $k_{2}, k_{4}, k_{5}$, and $k_{10}$ for generating $2 \mathrm{D}$ deformations of the geometric model, based on which images and in addition 2D displacement vector fields of actual material points are produced. The error norms used in comparing the ground truth vector field $\left(V_{g}\right)$ with the vector field measured by our warp 
algorithm $\left(V_{m}\right)$ are:

$$
\begin{gathered}
\varepsilon_{L}=\frac{1}{N} \sum|| V_{m}|-| V_{g}||, \\
\varepsilon_{\theta}=\frac{1}{\sum\left|V_{g}\right|} \sum\left|V_{g}\right| \cdot \arccos \frac{V_{g} \cdot V_{m}}{\left|V_{g}\right|\left|V_{m}\right|}
\end{gathered}
$$

where $\varepsilon_{L}$ measures the average difference in length between $V_{g}$ and $V_{m}$, and $\varepsilon_{\theta}$ measures the deviation in angle between $V_{g}$ and $V_{m} . \varepsilon_{\theta}$ requires further explanation. As can be seen from (12), we weigh individual angle deviations by the magnitude of the material point displacement vector; normalized by the sum of magnitude of all ground truth vectors. The reason for this is to emphasize angle deviation of points which have large displacements, and similarly to deemphasize the angle deviation of points which have a smaller displacement.

\begin{tabular}{|l|l|l|l|l|l|l|}
\hline TS & IP & $D_{0}$ & $T E$ & $T R$ & $T 1$ & $T 2$ \\
\hline $0.9 \mathrm{~cm}$ & $0.5 \mathrm{~cm}$ & 300 & $0.03 \mathrm{sec}$ & $10 \mathrm{sec}$ & $0.6 \mathrm{sec}$ & $0.1 \mathrm{sec}$ \\
\hline \hline$k_{x}$ & $k_{y}$ & $\theta$ & $R_{i}$ & $R_{o}$ & $\delta$ & $s s$ \\
\hline $7 \mathrm{rad} / \mathrm{cm}$ & $7 \mathrm{rad} / \mathrm{cm}$ & $45 \mathrm{deg}$. & 0.25 & 0.6 & $4 \mathrm{~cm}$ & $0.05 \mathrm{~cm} / \mathrm{pixel}$ \\
\hline \hline
\end{tabular}

Table 1. Imaging parameters and dimensions of geometric model. Please note that TS is tag separation, IP is the image plane position, $R_{i}$ and $R_{o}$ are the inner and outer radii of the 2 prolate spheroids, and $s s$ is the sample size.
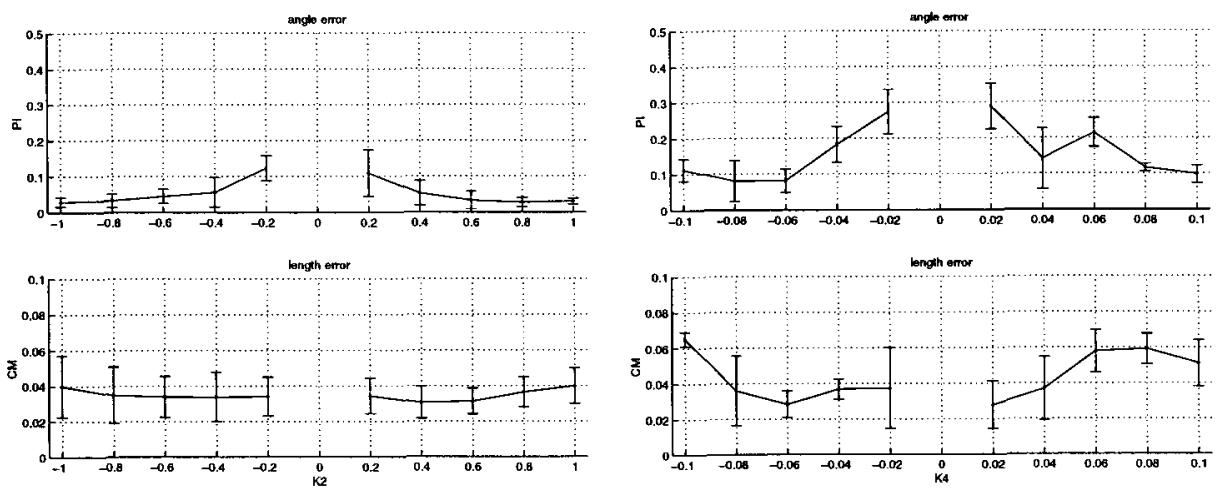

Fig. 1. The error plots for angle and length error for $k_{2}$ and $k_{4}$. Please see text for details.

Figures 1 and 2 show the angle and length errors by comparing $V_{m}$ and $V_{g}$ as a function of a range of values of $k_{2}, k_{4}, k_{5}$, and $k_{10}$, keeping the rest of the 

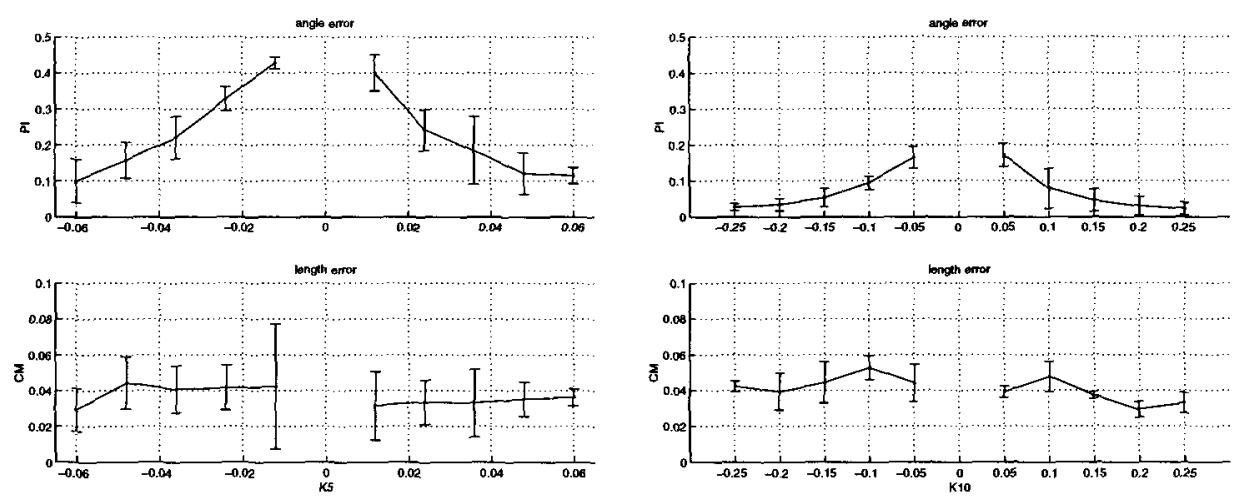

Fig. 2. The error plots for angle and length error for $k_{5}$ and $k_{10}$. Please see text for details.

$k$ parameters constant. The imaging and geometric parameters of the model for these experiments is shown in table 1. Additionally, as part of the validations and in order to test the sensitivity of the algorithms to different values of $\lambda_{1}, \lambda_{2}$, and $\lambda_{3}$, we varied each of these coefficients individually between the integers: 0 and 10, keeping the other 2 at the constant value of 1 (excluding $\lambda_{1}=0$ ). The error bars in these plots show the $3 \sigma$ range on either side of the error mean for particular values of each $k$ parameter. As can be seen from the figures, to a large degree the algorithm is insensitive to the exact values of $\lambda_{i}$ 's. An additional remarkable point regarding the error plots is the fact that for smaller motions, the value of $\varepsilon_{\theta}$ is larger than that for bigger motions. The reason for this unintuitive result can only be attributed to the larger percent inaccuracies in reconstruction of smaller displacements by the warping algorithm. Also, it should be noted that error plots in figures 1 and 2 subsume the errors incurred in localization of tags and myocardial contours (in this paper, tag and contour localization is performed through manual placement of control points of B-spline curves [2].) Although the magnitude of errors are bound to be smaller if accurate location of contour and tag lines in the simulated images were to be used, our complete system for tracking and reconstruction of tag lines would not be tested, and furthermore since the exact location of tags and contours are not known in real images, results may not be a good model of realistic situations.

Finally, Figure 3 displays true and reconstructed vector fields corresponding to torsion of the computational phantom.

\section{In-Vivo Validations}

Since "out-of-plane" movement of the LV occurs in slices close to the valveplane; i.e., at the top of the LV, for the purposes of extraction of $2 \mathrm{D}$ deformations, here we only consider SPAMM image slices acquired near the ventricular apex. Results of application of techniques to in vivo data of a normal human volunteer is shown in figure 4 . Note that in this case, the computed dense motion 

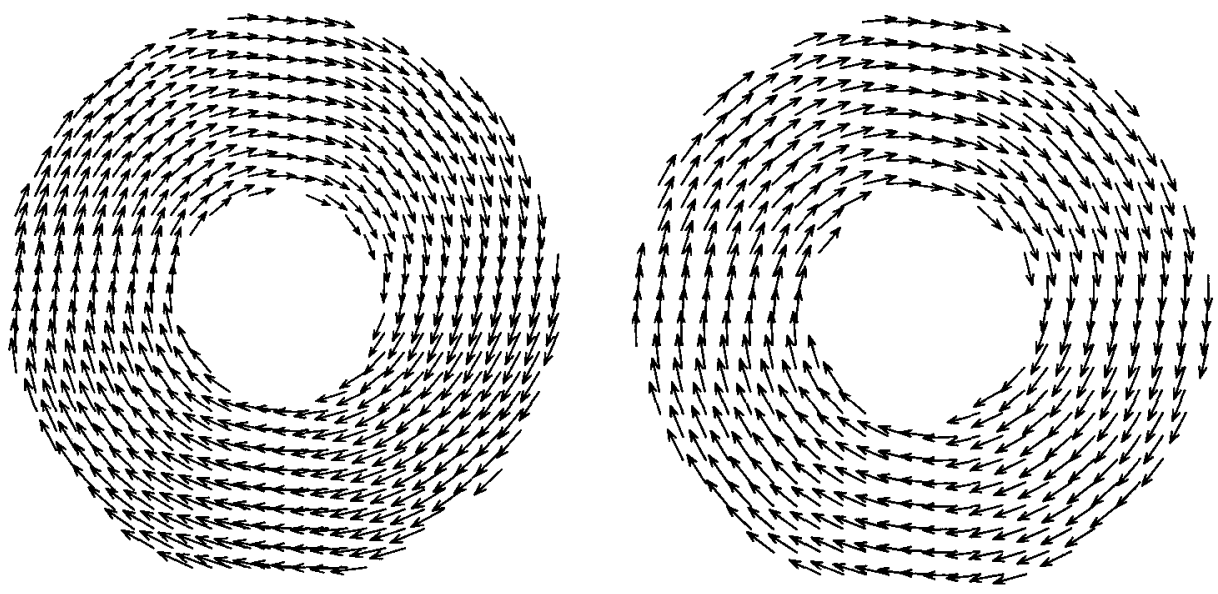

Fig. 3. Comparison of computed (left) and true (right) displacement vector fields corresponding to torsion $\left(k_{2}=-1.0\right)$.

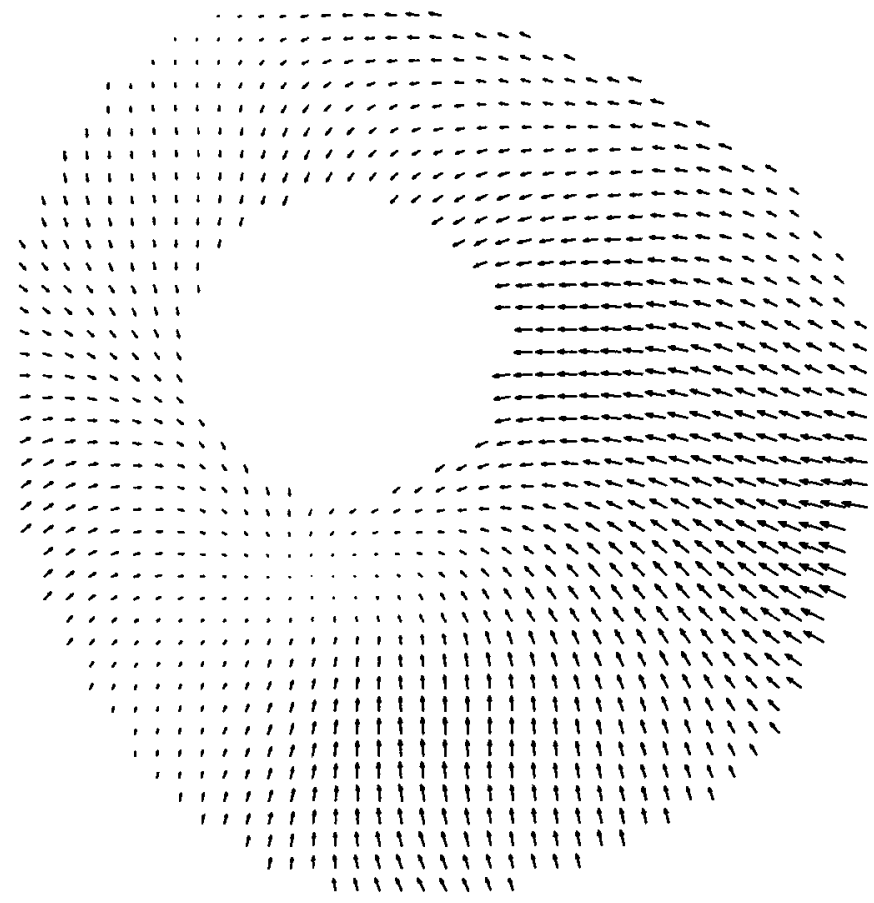

Fig. 4. The reconstructed vector field by CG computed from deforming a $7 \times 7 \mathrm{~B}$-spline grid for an apical slice acquired at 90 and $180 \mathrm{msec}$ after the ECG trigger. The vector field is displayed on the myocardial region in the $90 \mathrm{msec}$ image. 
is computed from two deformed grids. The endocardial and epicardial contours were each manually segmented through-out the sequence using a 6 control point $\mathrm{B}$-spline representation, as we have found automatic determination of myocardial contours in a SPAMM image sequence to be a formidable task due to the presence of tag lines. In order to assess the sensitivity of the vector field to different $\lambda_{i}$ coefficient values, the following study was undertaken. The vector field for the uniform weight factors $\lambda_{1}=1, \lambda_{2}=1$, and $\lambda_{3}=1$ was chosen as the ground-truth. Vector fields corresponding to different coefficient values were subsequently compared with this vector field. Results are illustrated in table 2 .

\section{Conclusions}

We have described new methods for efficient reconstruction of dense displacement vector fields from SPAMM grids. The constrained thin-plate spline methods warp an area in the plane such that two embedded grids of curves are non-rigidly registered, thereby interpolating a dense displacement vector field. The new warp method treats intersection points of SPAMM grids as standard landmarks and forces these to come together. Furthermore, it corresponds complete tag curves and brings these into alignment. Finally, where no information is available, it interpolates a $C^{1}$ continuous vector field.

In addition to the developed machinery in this paper, methods were evaluated using a cardiac motion simulator. The methods have been tested for accuracy in length as well as angle of the reconstructed displacement vectors from the known ground-truth, and the results indicate that constrained thin-plate spline reconstructions of myocardial deformations is sufficiently accurate for measurement of in-plane tissue deformations within a reasonable time and between any two frames in a sequence of tagged images.

\begin{tabular}{|l|l|l|l|l|}
\hline$\lambda_{1}$ & $\lambda_{2}$ & $\lambda_{3}$ & Angle Error & Length Error \\
\hline \hline 1 & 1 & 1 & 0 & 0 \\
\hline 5 & 1 & 1 & 0.006 & 0.019 \\
\hline 10 & 1 & 1 & 0.008 & 0.029 \\
\hline 1 & 5 & 1 & 0.005 & 0.012 \\
\hline 1 & 10 & 1 & 0.006 & 0.015 \\
\hline 1 & 1 & 5 & 0.005 & 0.015 \\
\hline 1 & 1 & 10 & 0.008 & 0.021 \\
\hline 1 & 1 & 0 & 0.007 & 0.018 \\
\hline 1 & 0 & 1 & 0.011 & 0.043 \\
\hline
\end{tabular}

Table 2. The values for angle (fraction of $\pi$ ) and length errors (in $\mathrm{cm}$ ) as a function of $\lambda_{1}, \lambda_{2}$, and $\lambda_{3}$. The "ground-truth" in this case was chosen to be the reconstructed displacement field with $\lambda_{i}=1$. 


\section{References}

1. A. A. Amini. Automated methods for cardiac motion analysis from MR tagging. Proposal funded by The Whitaker Foundation, 1992.

2. A. A. Amini, R. W. Curwen, and John C. Gore. Snakes and splines for tracking non-rigid heart motion. In European Conference on Computer Vision, pages 251261, University of Cambridge, UK, April 1996.

3. A. A. Amini and et al. MR physics-based snake tracking and dense deformations from tagged MR cardiac images (oral presentation). In AAAI Symposium on Applications of Computer Vision to Medical Image Processing, Stanford University, Stanford, California, March 1994.

4. T. Arts, W. Hunter, A. Douglas, A. Muijtjens, and R. Reneman. Description of the deformation of the left ventricle by a kinematic model. J. Biomechanics, 25(10):1119-1127, 1992.

5. L. Axel and L. Dougherty. MR imaging of motion with spatial modulation of magnetization. Radiology, 171(3):841-845, 1989.

6. F. Bookstein. Principal warps: Thin-plate splines and the decomposition of deformations. IEEE Transactions on Pattern Analysis and Machine Intelligence, PAMI-11:567-585, 1989.

7. T. Denney and J. Prince. Reconstruction of 3-d left ventricular motion from planar tagged cardiac MR images: An estimation-theoretic approach. IEEE Transactions on Medical Imaging, 14(4):625-635, December 1995.

8. W. Grimson. An implementation of a computational theory of visual surface interpolation. Computer Vision, Graphics, and Image Processing, 22:39-69, 1983.

9. J. Park, D. Metaxas, and L. Axel. Volumetric deformable models with parameter functions: A new approach to the 3d motion analysis of the LV from MRI-SPAMM. In International Conference on Computer Vision, pages 700-705, 1995.

10. W. Press, B. Flannery, S. Teukolsky, and W. Vetterling. Numerical recipes in $C$. Cambridge University Press, Cambridge, 1988.

11. P. Radeva, A. Amini, and J. Huang. Deformable B-Solids and implicit snakes for $3 \mathrm{~d}$ localization and tracking of SPAMM MRI data. Computer Vision and Image Understanding, 66(2):163-178, May 1997.

12. D. Terzopoulos. Multiresolution Computation of Visible Representation. PhD thesis, MIT, 1984.

13. E. Waks, J. Prince, and A. Douglas. Cardiac motion simulator for tagged MRI. In Proc. of Mathematical Methods in Biomedical Image Analysis, pages 182-191, 1996.

14. A. Young, D. Kraitchman, L. Dougherty, and L. Axel. Tracking and finite element analysis of stripe deformation in magnetic resonance tagging. IEEE Transactions on Medical Imaging, 14(3):413-421, September 1995.

15. E. Zerhouni, D. Parish, W. Rogers, A. Yang, and E. Shapiro. Human heart: Tagging with MR imaging - a method for noninvasive assessment of myocardial motion. Radiology, 169:59-63, 1988. 